\title{
Intensity noise as a driver for transverse mode instability in fiber amplifiers
}

\author{
Christoph Stihler ${ }^{1,2^{*}}$ (D), Cesar Jauregui ${ }^{1}$, Sobhy E. Kholaif ${ }^{1,3}$ and Jens Limpert ${ }^{1,2,3}$
}

\author{
* Correspondence: christoph. \\ stihler@uni-jena.de \\ ${ }^{1}$ Institute of Applied Physics, Abbe \\ Center of Photonics, \\ Friedrich-Schiller-Universität Jena, \\ Albert-Einstein-Str. 15, 07745 Jena, \\ Germany \\ ${ }^{2}$ Fraunhofer Institute for Applied \\ Optics and Precision Engineering, \\ Albert-Einstein-Str. 7, 07745 Jena, \\ Germany \\ Full list of author information is \\ available at the end of the article
}

\begin{abstract}
The effect of transverse mode instability (TMI) is currently the main limitation for the further average-power scaling of fiber laser systems with diffraction-limited beam quality. In this work a main driving force for TMI in fiber amplifiers is identified. Our experiments and simulations illustrate that the performance of fiber laser systems in terms of their diffraction-limited output power can be significantly reduced when the pump or seed radiation exhibit intensity noise. This finding emphasizes the fact that the TMI threshold is not only determined by the active fiber but, rather, by the whole system. In the experiment an artificially applied pump intensity-noise of $2.9 \%$ led to a reduction of the TMI threshold of $63 \%$, whereas a similar seed intensitynoise decreased it by just $13 \%$. Thus, even though both noise sources have an impact on the TMI threshold, the pump intensity-noise can be considered as the main driver for TMI in saturated fiber amplifiers. Additionally, the work unveils that the physical origin of this behavior is linked to the noise transfer function in saturated fiber amplifiers. With the gained knowledge and the experimental and theoretical results, it can be concluded that a suppression of pump-noise frequencies below $20 \mathrm{kHz}$ could strongly increase the TMI threshold in high-power fiber laser systems.
\end{abstract}

Keywords: Transverse mode instability, Fiber lasers, Thermal effects, Intensity noise , Modal interference, Refractive index grating, Mode coupling, Phase shift

\section{Introduction}

Fiber laser systems are able to simultaneously deliver high average powers and excellent beam quality. This is mostly because they can handle high heat loads due to the efficient heat extraction granted by their large surface-to-volume ratio [1, 2]. Despite that, even the fiber-laser technology has suffered from thermal effects during the last decade. The most detrimental of these effects is transverse mode instability (TMI), which discovery was first published in 2010 [3]. TMI manifests itself as a dynamic energy transfer between different transverse modes, which occurs sharply above a specific average-power threshold [4]. As a result, above this TMI threshold the beam profile exhibits spatial and temporal fluctuations on a millisecond timescale [5], which prevent the utilization of fiber lasers and amplifiers for many applications.

The physical origin of TMI is linked to a modal interference pattern (MIP) that is generated by at least two transverse fiber modes, which have different propagation constants [6]. Due to heat sources such as the quantum defect and photodarkening,

(c) The Author(s). 2020 Open Access This article is distributed under the terms of the Creative Commons Attribution 4.0 International License (http://creativecommons.org/licenses/by/4.0/), which permits unrestricted use, distribution, and reproduction in any medium, provided you give appropriate credit to the original author(s) and the source, provide a link to the Creative Commons license, and indicate if changes were made. 
the MIP induces a quasi-periodic temperature pattern along the fiber, which eventually creates a thermally-induced refractive index grating (RIG) due to the thermooptic effect. Such a RIG has the ability to potentially couple energy between different modes, in a similar way to long-period transmission gratings [7]. For a modal energy transfer to actually take place, however, it is necessary that the MIP and the RIG are longitudinally shifted with respect to one another, i.e. a phase shift must occur [8]. Recently, it was revealed that such a phase shift between the MIP and the RIG can be induced by a pump-power change [9]. The reason for the generation of this phase shift is the variation of the extracted power due to the pump-power change, which causes a transient heat-load change in the fiber. This heat-load change eventually modifies the refractive index profile of the waveguide and, herewith, the propagation constants of the modes, which leads to a longitudinal shift of the MIP. Finally, a phase shift between the MIP and the RIG develops, since the RIG cannot follow the MIP instantaneously because it needs time to evolve from its former position [9]. Experimental investigations have supported this theoretical statement by demonstrating that pump-power-induced phase shifts can lead to a modal energy transfer even below the TMI threshold [10]. This finding indicates that such a phase shift is most likely the trigger for TMI. Furthermore, the experiments in [10] have shown that the fiber becomes more and more sensitive to phase shifts the higher the average output power is. Thus, it was concluded that even extremely small phase shifts are sufficient to provoke TMI at the threshold power. In this context, it is possible that the intensity noise of the pump radiation functions as a driving force for TMI, since it can induce small phase shifts between the MIP and the RIG. Similarly, the intensity noise of the seed radiation can generate phase shifts via a modulation of the extracted power and, thus, of the heat load in a fiber amplifier.

In the past, the impact of pump and seed intensity-noise on TMI has only been studied theoretically [11-14]. Due to the different results obtained in the different studies and due to the lack of physical explanations, it is not clear so far which noise source affects the TMI threshold of fiber amplifiers the most: the pump or the seed intensitynoise. However, this knowledge is essential to further scale the output power of diffraction-limited fiber laser systems. Thus, the aim of this work is to unveil the main driver for TMI by comparing the impact of pump and seed intensity-noise on the TMI threshold of a fiber amplifier, both experimentally and theoretically. Additionally, the physics behind the observed dependences will be revealed and, with the gained knowledge, a guideline to increase the TMI threshold of high-power fiber laser systems will be provided.

\section{Methods}

\section{Noise generation and experimental setup}

To investigate the impact of noise on the TMI threshold, we have artificially imprinted noise onto the pump and seed radiation, respectively. In order to do this, we have generated white noise with a computer, since it represents a homogeneous distribution of the noise energy among all frequencies. Afterwards, this white noise has been transferred, via an arbitrary waveform generator (AWG, Rigol DG4202), to the seed or the pump of the fiber amplifier. 
In case of the pump intensity-noise, the AWG was connected to the driver (Delta Elektronika SM 120-50) of the pump laser-diode (Dilas D4F4Q22-976.3-2000CIS45.8), which pumped the main-amplifier fiber at $976 \mathrm{~nm}$ in a counter-propagating configuration, as can be seen in Fig. 1 (blue dashed box). The main-amplifier fiber was an Yb-doped Large-Pitch Fiber (LPF) [15] with a core diameter of $\sim 65 \mu \mathrm{m}$, a similar mode-field diameter and a length of $1.07 \mathrm{~m}$. To measure the pump intensity-noise, a small reflection of the pump beam was directed to a photodiode (Thorlabs PDA20CSEC, blue solid box), which was terminated with $50 \Omega$ and connected to a 12 bit oscilloscope (Teledyne Lecroy HDO6104). The noise was acquired during $10 \mathrm{~s}$ with a sample rate of $10^{6}$ samples/s and a $240 \mathrm{kHz}$ low-pass filter was used to avoid aliasing. Before the experiments were carried out, the noise trace was optimized with the help of the photodiode signal, in order to get a spectrum as flat as possible. This procedure allowed us to pre-compensate for any spectrally-dependent noise attenuation, which could be introduced by the electronics of the driver and/or the laser diode.

In order to transfer the noise to the seed signal, the AWG modulated the driver (Delta Elektronika SM 120-50) of the laser diode that pumped the pre-amplifier fiber, as depicted in Fig. 1 (black dashed box). This, in turn, resulted in a modulation of the seed radiation of the main amplifier fiber. The active fiber was seeded by a source delivering fs-pulses (stretched to $1 \mathrm{~ns}$ ) with a repetition rate of $19 \mathrm{MHz}$ and an average signal power of $5 \mathrm{~W}$ centered at $1030 \mathrm{~nm}$ (with a $3 \mathrm{~dB}$-bandwidth of $7 \mathrm{~nm}$ ). Finally, the leakage through one of the input-coupling mirrors was used to measure the seed intensity-noise with the same photodiode (black solid box) that was used for the pumpnoise measurements.

\section{Frequency region of interest}

Former investigations on TMI have revealed that the beam fluctuations (which are related to the modal energy transfer) close to the TMI threshold occur at a dominant frequency typically below $10 \mathrm{kHz}$ [5]. This frequency depends on the fiber design and, particularly, on the mode-field diameter and is related to the thermal diffusion time of the fiber core. Thus, it is expected that some noise frequencies will have a stronger impact on the TMI threshold than others. To verify this, noise traces with different

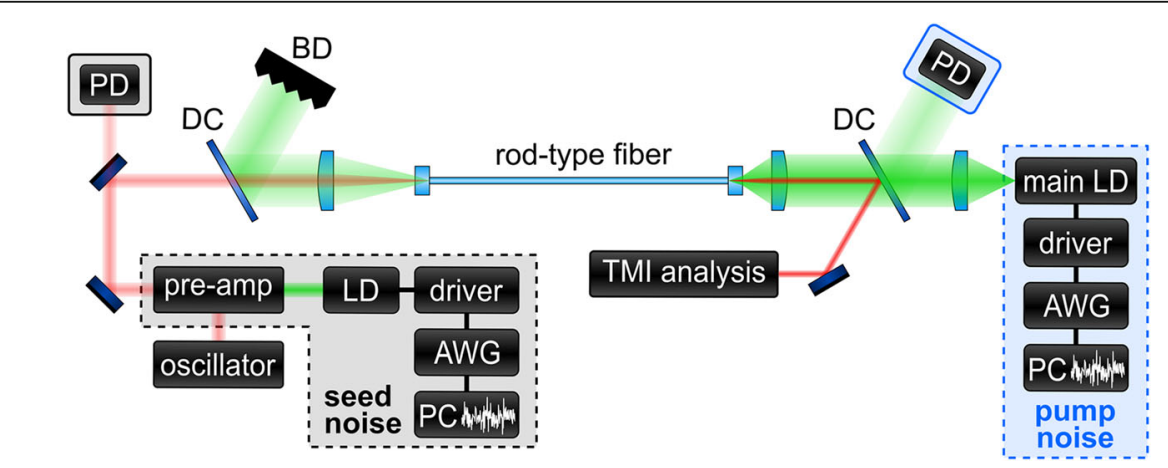

Fig. 1 Experimental setup. Pump-noise generation (blue dashed box), seed-noise generation (black dashed box), pump-noise measurement (blue solid box), seed-noise measurement (black solid box); AWG - arbitrary waveform generator, BD - beam dump, DC - dichroic mirror, LD - laser diode, PC - personal computer, PD - photodiode 
bandwidths have been generated and imprinted onto the pump and seed radiation, respectively, and the corresponding TMI threshold was measured.

\section{Pump intensity-noise}

To determine the frequency region of interest, white noise was first generated from $1 \mathrm{~Hz}$ up to a cut-off frequency of $6 \mathrm{kHz}$. This frequency band contains all relevant frequencies of the TMI-induced beam fluctuations for the active fiber used in the experiments, which typically lie below $2 \mathrm{kHz}$. To generate noise traces with different bandwidths, the cut-off frequency was progressively decreased, which means that all frequency components above this cut-off value were deleted from the trace. By doing this, noise traces with cut-off frequencies from $6 \mathrm{kHz}$ down to $75 \mathrm{~Hz}$ were created. All these noise traces were then imprinted onto the pump radiation consecutively and the corresponding TMI thresholds were measured according to the guidelines and definitions in [5].

Figure 2 shows selected examples of the pump-noise traces measured with the photodiode (blue solid box in Fig. 1). Their power spectral density is depicted in Fig. 2a and the integrated noise (RIN - relative intensity noise) is shown in Fig. 2b. The RIN was calculated by integrating the power spectral density from $500 \mathrm{kHz}$ (given by the sample rate of the oscilloscope) down to $1 \mathrm{~Hz}$ (determined by the acquisition time) for each measurement.

It is important to stress that the power per frequency segment was kept constant when reducing the bandwidth of the noise trace. This resulted in the same powerspectral-density distribution up to the respective cut-off frequency in each trace (see Fig. 2a). That also implies that the integrated noise (i.e. the RIN) becomes smaller the lower the cut-off frequency is (see Fig. 2b) (since the overall noise energy is reduced). For ideal white noise, the RIN in Fig. $2 \mathrm{~b}$ should follow a linear behavior since each

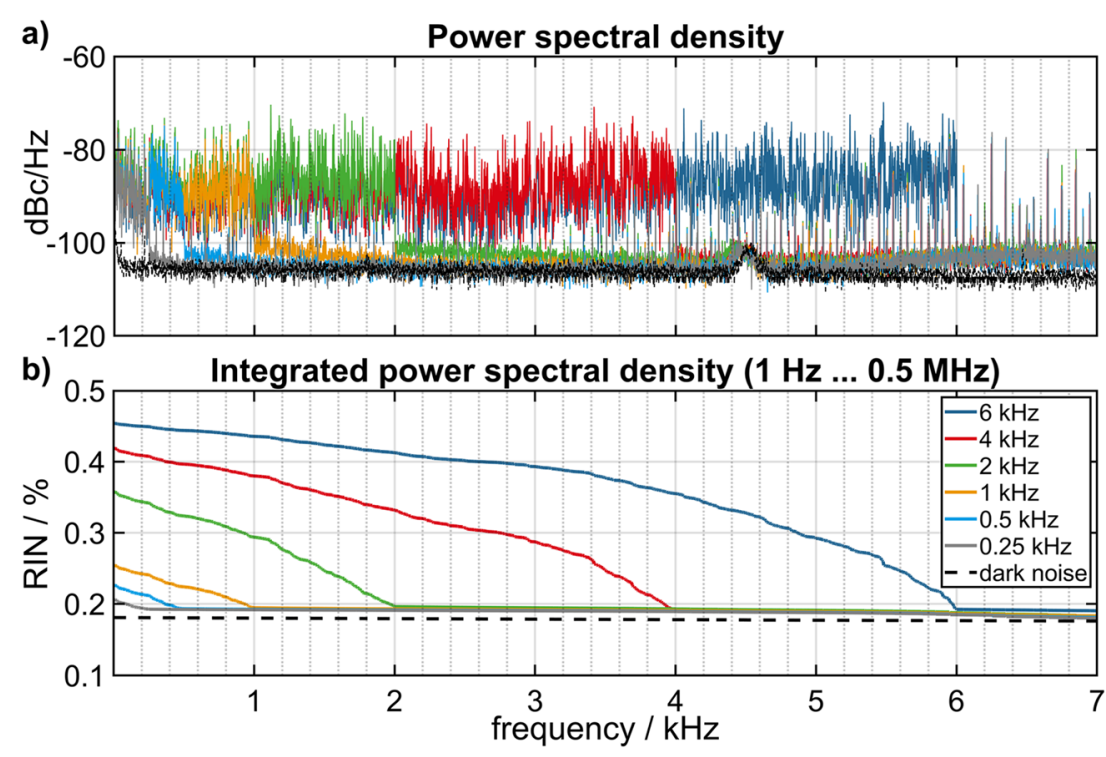

Fig. 2 Measured pump intensity-noise with different cut-off frequencies. a Power spectral density and $\mathbf{b}$ Relative intensity noise (RIN: integrated power spectral density from $500 \mathrm{kHz}$ down to $1 \mathrm{~Hz}$; the frequency range for the integration is determined by the sample rate of the oscilloscope and by the acquisition time) 
frequency noise component should contribute equally to the integrated noise. As can be seen, we have not been able to fully achieve this behavior, since, even though we optimized the noise traces, some frequency components are slightly more pronounced than others. Nevertheless, especially for lower cut-off frequencies (which will become more important in the following), the dependence of the RIN on the noise frequency approaches a linear function.

A reduction of the TMI threshold has been observed when imprinting the noise traces onto the pump radiation. The amplitude of the pump intensity-noise, which can be inferred from Fig. 2, was chosen in such a way that the broadest noise trace (with a cut-off frequency $6 \mathrm{kHz}$ ) caused a reduction of the TMI threshold sufficiently strong to extract a clear statement from the subsequent measurements.

The decrease of the TMI threshold changed depending on which frequencies components were contained in the pump intensity-noise, as can be seen in Fig. 3. When imprinting the pump-noise trace with frequency components from $1 \mathrm{~Hz}$ up to $6 \mathrm{kHz}$ (cutoff frequency $=6 \mathrm{kHz}, \mathrm{RIN}=0.455 \%$ ), the TMI threshold was reduced from the initial $260 \mathrm{~W}$ (no artificial noise, red circle) to a value of $211 \mathrm{~W}$. A similar decrease of the TMI threshold was observed for cut-off frequencies of $4 \mathrm{kHz}$ and $2 \mathrm{kHz}$. This finding implies that pump-noise frequencies above $2 \mathrm{kHz}$ have no significant influence on the TMI threshold of the used fiber (indicated by the red shading in Fig. 3).

However, when decreasing the cut-off frequency further and, thus, cutting more of the high pump-noise frequencies, the reduction of the TMI threshold became less pronounced. This means that the frequencies that have been cut, were responsible for the former reduction of the TMI threshold. This trend continues for cut-off frequencies of a few hundreds of $\mathrm{Hz}$ until it saturates below $100 \mathrm{~Hz}$. In general, it can be said that pump-noise frequencies below $2 \mathrm{kHz}$ have an impact on the TMI threshold of the used

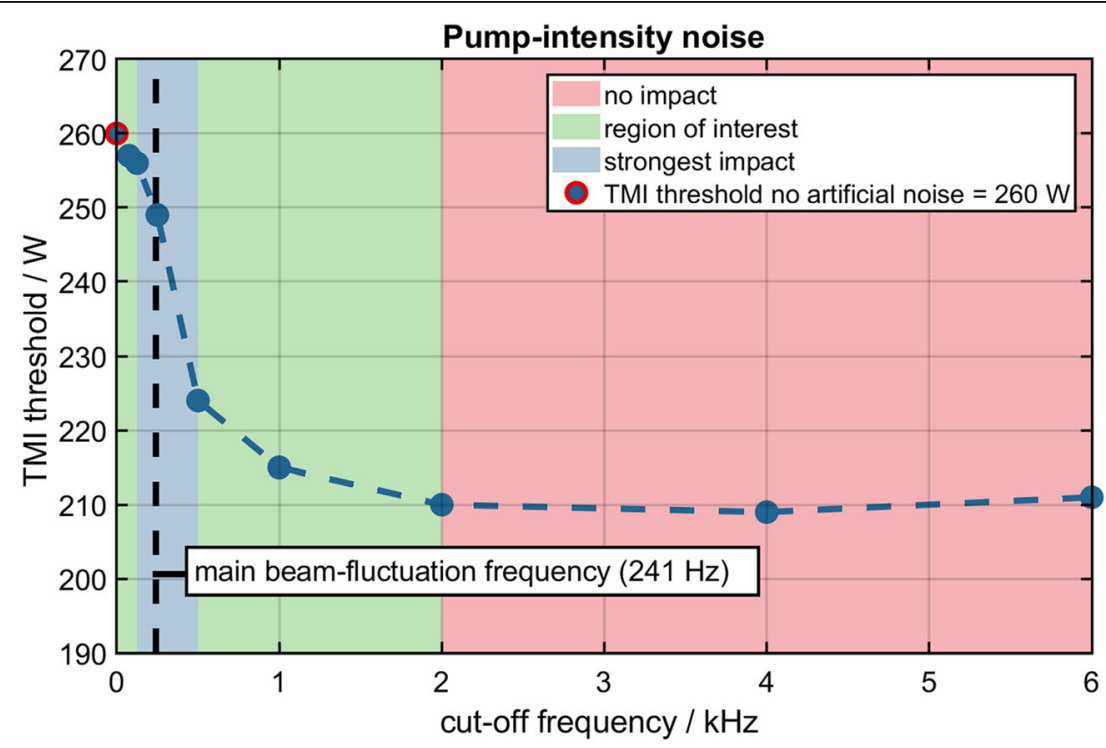

Fig. 3 Dependence of the TMI threshold on the frequency bandwidth of the pump intensity-noise. The applied noise traces range from $1 \mathrm{~Hz}$ up to the displayed cut-off frequencies (detailed in Fig. 2). Pump-noise frequencies between $125 \mathrm{~Hz}$ and $500 \mathrm{~Hz}$ have the strongest impact on the TMI threshold (blue shading), which corresponds to the main beam-fluctuation frequency of $241 \mathrm{~Hz}$ (black dashed line; measured slightly above the TMI threshold). In a more general way, it can be seen that only frequencies below $2 \mathrm{kHz}$ influence the TMI threshold of the used active fiber (green shading) 
active fiber and, thus, this frequency band represents the region of interest (green shading in Fig. 3) for the subsequent investigations. Hence, the noise frequencies that influence the TMI threshold seem to be similar to the frequencies of the beam fluctuations during TMI. This statement will be confirmed in the next paragraph. Please note that the frequency region of interest will differ from fiber to fiber since it is related to the design parameters, such as e.g. the mode-field diameter. However, the frequency region of interest will typically not exceed $20 \mathrm{kHz}$ even in high-power fiber amplifiers with smaller mode-field diameters (e.g. $20 \mu \mathrm{m}$ ), since all TMI-related beam fluctuations in fiber amplifiers reported so far occurred with frequencies below $20 \mathrm{kHz}[5,16-18]$.

For the active fiber used in these experiments, the pump-noise frequencies between $125 \mathrm{~Hz}$ and $500 \mathrm{~Hz}$ have the strongest impact on the TMI threshold (indicated by the blue shading in Fig. 3). This corresponds to the main beam-fluctuation frequency of $241 \mathrm{~Hz}$ in the active fiber, which was measured slightly above the TMI threshold according to [5]. Thus, noise frequencies around the main beam-fluctuation frequency of a fiber most likely induce the strongest phase shift between the MIP and the RIG, which results in a strong modal energy transfer. This is because the phase-shift introduction is linked to a heat-load change and, thus, directly to the thermal diffusion time of the fiber [9].

\section{Seed intensity-noise}

The same investigations have been done to determine the frequency region of interest for the seed intensity-noise. Therefore, exactly the same noise traces as before were imprinted onto the pump radiation of the pre-amplifier, which have then been converted into intensity noise of the seed radiation for the main amplifier. This noise was measured with the photodiode (black solid box in Fig. 1) placed behind one of the

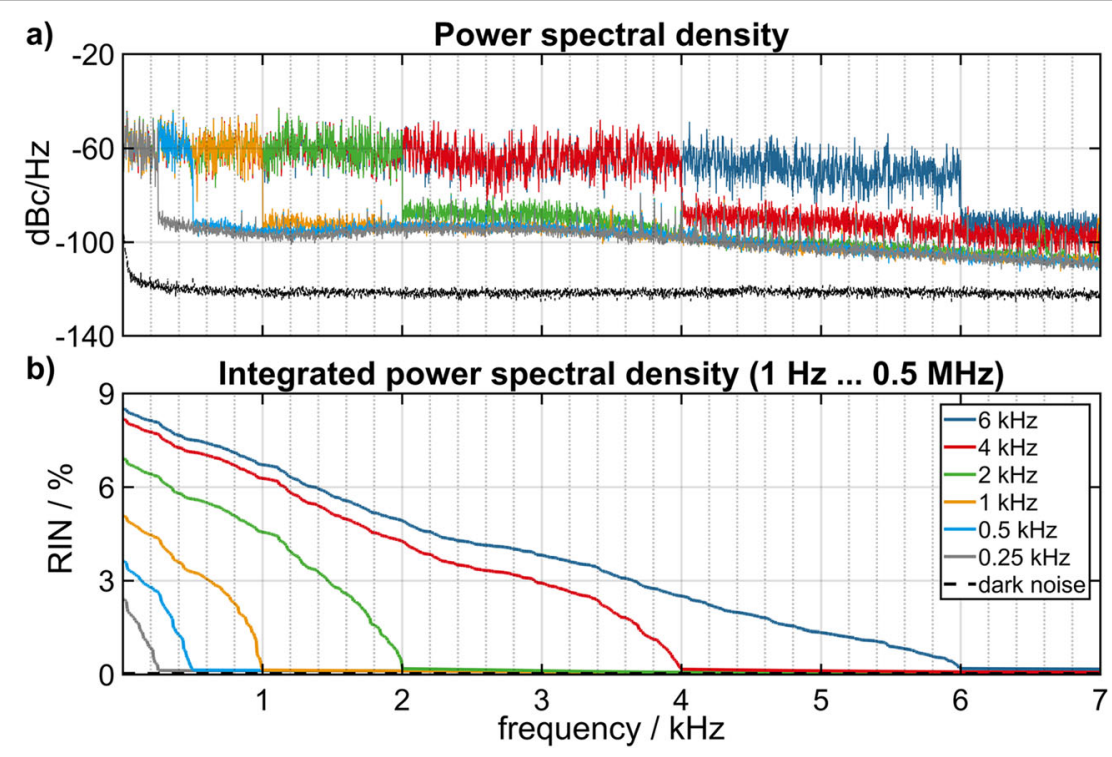

Fig. 4 Measured seed intensity-noise with different cut-off frequencies. a Power spectral density and $\mathbf{b}$ Relative intensity noise (RIN: integrated power spectral density from $500 \mathrm{kHz}$ down to $1 \mathrm{~Hz}$; the frequency range for the integration is determined by the sample rate of the oscilloscope and by the acquisition time) 


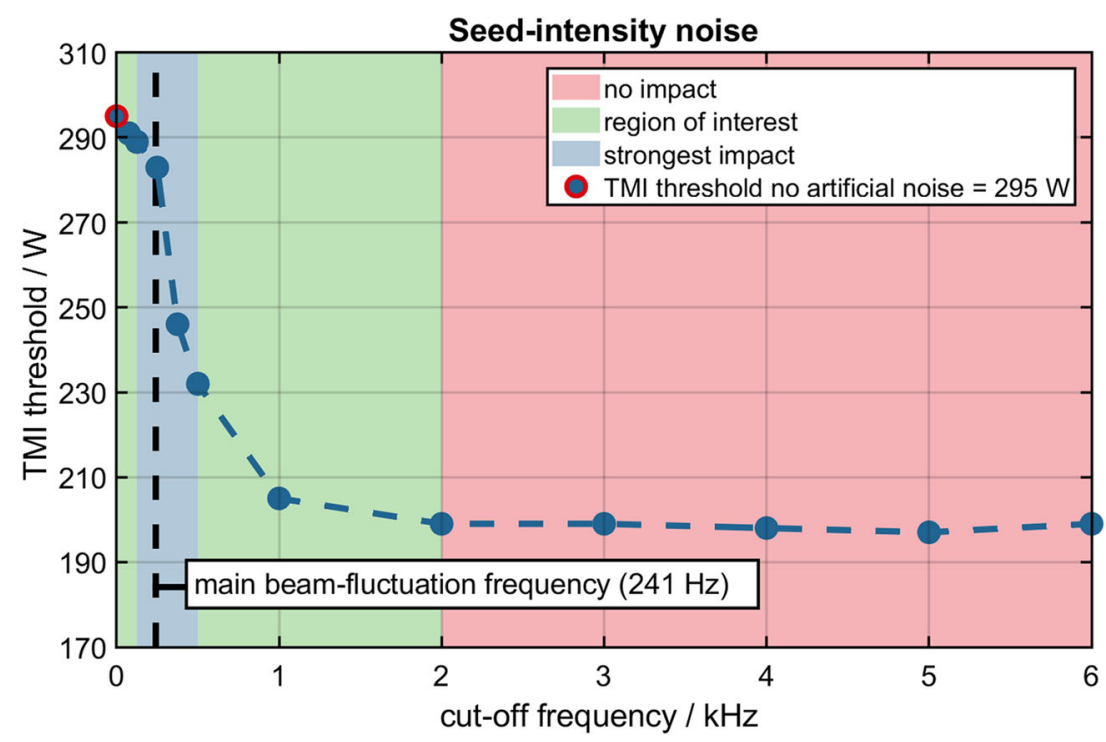

Fig. 5 Dependence of the TMI threshold on the frequency bandwidth of the seed intensity-noise. The observed behavior is similar to the one obtained for the pump intensity-noise (see Fig. 3)

input-coupling mirrors. Selected pump-noise traces are depicted in Fig. 4, where the power spectral density is illustrated in Fig. 4a and the RIN (integrated from $500 \mathrm{kHz}$ down to $1 \mathrm{~Hz}$ ) is shown in Fig. 4b. Note that the traces in Fig. 4a have inconsistent noise floors above their corresponding cut-off frequency, which was caused by an imperfect noise imprinting. However, this did not influence the subsequent measurements since the noise floor of each trace was still $\sim 30 \mathrm{~dB}$ below its imprinted signal.

As in the previous experiment, the applied noise resulted in a decrease of the TMI threshold, which was initially measured to be $295 \mathrm{~W}$ (no artificial noise). Note that the initial TMI thresholds of the pump- and the seed-noise measurement differ slightly, which is most likely due to maintenance-related adjustments in between the measurements.

The measured TMI thresholds as a function of the cut-off frequency of the corresponding seed-noise trace are illustrated in Fig. 5 and show a behavior similar to that of the pump-noise investigations. Consequently, it can be concluded that also seednoise frequencies above $2 \mathrm{kHz}$ have no significant impact on the TMI threshold of the used active fiber (red shading in Fig. 5), whereas frequencies below $2 \mathrm{kHz}$ result in a decrease of the TMI threshold (green shaded area in Fig. 5). Similar to the pumpintensity-noise case, seed-noise frequencies between $125 \mathrm{~Hz}$ and $500 \mathrm{~Hz}$ have the strongest influence on the TMI threshold (blue shaded area in Fig. 5), which corresponds to the main beam-fluctuation frequency of the fiber.

To double-check the results of the frequency dependence of pump and seed intensity-noise, a trace with noise content contained only within a narrow frequency band was generated. The frequency band of the noise trace has then been tuned across the interesting frequency region and the corresponding TMI thresholds have been measured. The results obtained in this experiment have confirmed the conclusions extracted from Figs. 3 and 5. 


\section{Experimental results and discussion}

As detailed in "Frequency region of interest" section, the frequency region of interest for both pump and seed intensity-noise was found to be in the range from $1 \mathrm{~Hz}$ up to $2 \mathrm{kHz}$ for the active fiber used in these experiments. Thus, to investigate the impact of both noise sources on the TMI threshold, the same noise trace with a cut-off frequency of $2 \mathrm{kHz}$ was taken and its amplitude was changed. The generated noise traces were then imprinted onto the pump or seed radiation, depending on the experiment. Using this procedure, the noise has been investigated with the photodiodes, as illustrated in Fig. 1, and the RIN has been calculated. Thus, the higher the amplitude of the noise trace, the larger its RIN is. This way the RIN becomes a measure for the strength of the applied noise, which was used for the subsequent investigations.

Additionally, the corresponding TMI thresholds have been determined according to [5]. This measurement procedure was developed in a way that the TMI threshold only depends on the change of the stability and not on the initial stability of the fiber laser system (i.e. in our case the applied pump/seed noise), which is an essential feature for the experiments described in this paper.

The general observation in these experiments is that, when applying artificial pump or seed intensity-noise, the TMI threshold was decreased, as will be detailed later. Thus, the stronger the noise was, the lower the average power at which the beam suffered from fluctuations. Interestingly, with low pump-/seed-noise amplitudes the beam fluctuations had a steeper dependence on the average power, whereas with higher pump-/seed-noise levels the strength of the fluctuations increased in a smoother way when increasing the average power. Thus, the transition region of TMI became broader with increasing pump-/seed-noise levels. This finding is object of current investigations and will not be further detailed here. However, this observation does not influence the findings of this work, since the threshold condition detailed in [5] is not affected by it.

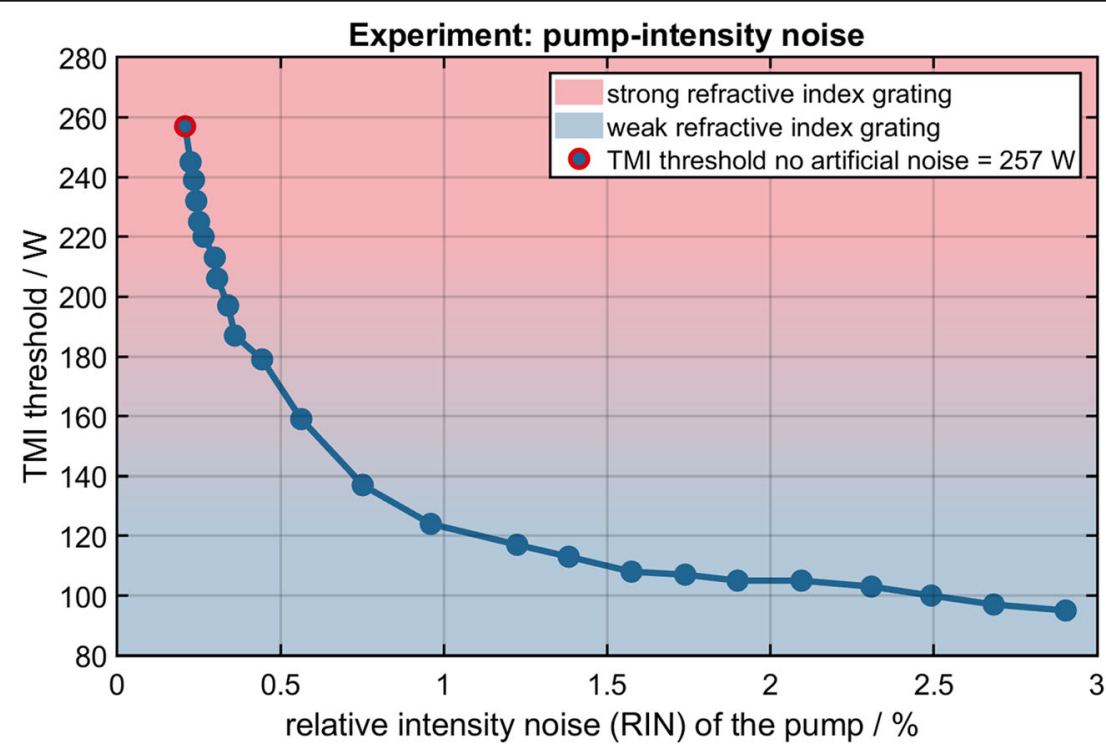

Fig. $6 \mathrm{TMI}$ threshold as a function of the relative intensity noise (RIN) of the pump. The artificial pump intensity-noise contains frequencies from $1 \mathrm{~Hz}$ up to $2 \mathrm{kHz}$. For a value of $2.9 \%$ the pump-RIN decreases the TMI threshold by $63 \%$ from its intrinsic value (95 W vs. $257 \mathrm{~W}$ ) 
The next two subsections will discuss the impact of pump and seed intensity-noise on the TMI threshold of a high-power fiber amplifier.

\section{Pump intensity-noise}

Figure 6 illustrates the TMI threshold as a function of the pump-RIN. As can be seen, the pump intensity-noise has a substantial impact on the TMI threshold of the fiber amplifier. By applying a pump intensity-noise of only $2.9 \%$ (from $1 \mathrm{~Hz}$ up to $2 \mathrm{kHz}$ ) the TMI threshold was decreased down to $95 \mathrm{~W}$, which corresponds to a reduction of $63 \%$ from the intrinsic value of $257 \mathrm{~W}$ (no artificial noise, RIN $=0.208 \%$ ). This result becomes even more significant when taking into account that only a narrow frequency range $(125 \ldots 500 \mathrm{~Hz})$ is responsible for the major part of the decrease of the TMI threshold. Therefore, if the noise would only occupy this narrow frequency range, the RIN required to obtain the same reduction of the TMI threshold would be even lower (by roughly a factor $\sim 5$ ).

As illustrated in Fig. 6 the TMI threshold experiences a non-linear decrease with increasing pump-RIN. In fact, when imprinting a pump intensity-noise slightly stronger than the intrinsic value of $0.208 \%$ (no artificial noise), the threshold drops rapidly. However, the same increase of pump-RIN at higher pump-noise amplitudes leads to a significantly weaker decrease of the TMI threshold. This behavior can be understood when taking into account the sensitivity of the refractive index grating (RIG) to phase shifts at different average output powers [10]. As mentioned above, two requirements need to be fulfilled to enable a modal energy transfer in a fiber: a sufficiently strong RIG and a phase shift between the MIP and the RIG. Hence, a similar modal energy transfer can be achieved either with a strong RIG and a small phase shift or, alternatively, with a weak RIG and a large phase shift. In more detail, it has been recently revealed, that the strength of the RIG in high-power fiber laser systems increases linearly with the average output power, whereas the modal energy transfer increases nonlinearly [10]. This means that the RIG becomes more and more sensitive to small phase shifts with increasing average output power. The consequence of this finding for the experimental results depicted in Fig. 6 is that, since at high average output powers the RIG is strong (red shaded region in Fig. 6), a small phase shift (i.e. low RIN) is sufficient to induce a modal energy transfer and, thus, to strongly decrease the TMI threshold. To reach the same decrease of the TMI threshold (and, thus, to cause the same energy transfer) at low average powers, a much larger phase shift (i.e. high RIN) is needed, because at low average powers the RIG is weaker (blue shaded region in Fig. 6) and not so sensitive to phase shifts.

From Fig. 6 (i.e. by extrapolating the TMI threshold in the region of low pump-RIN) it can be expected that a reduction of the intrinsic pump intensity-noise will lead to a steep increase of the TMI threshold. This threshold increase will most likely continue until other noise sources become more dominant in terms of the phase shift they induce.

In conclusion, the experimental investigations have shown that pump intensity-noise can significantly reduce the performance of fiber amplifiers in terms of their diffraction-limited output power. Thus, in order to reach high TMI thresholds, it is essential to have both a pump laser-diode and a laser driver which possess low noise in 


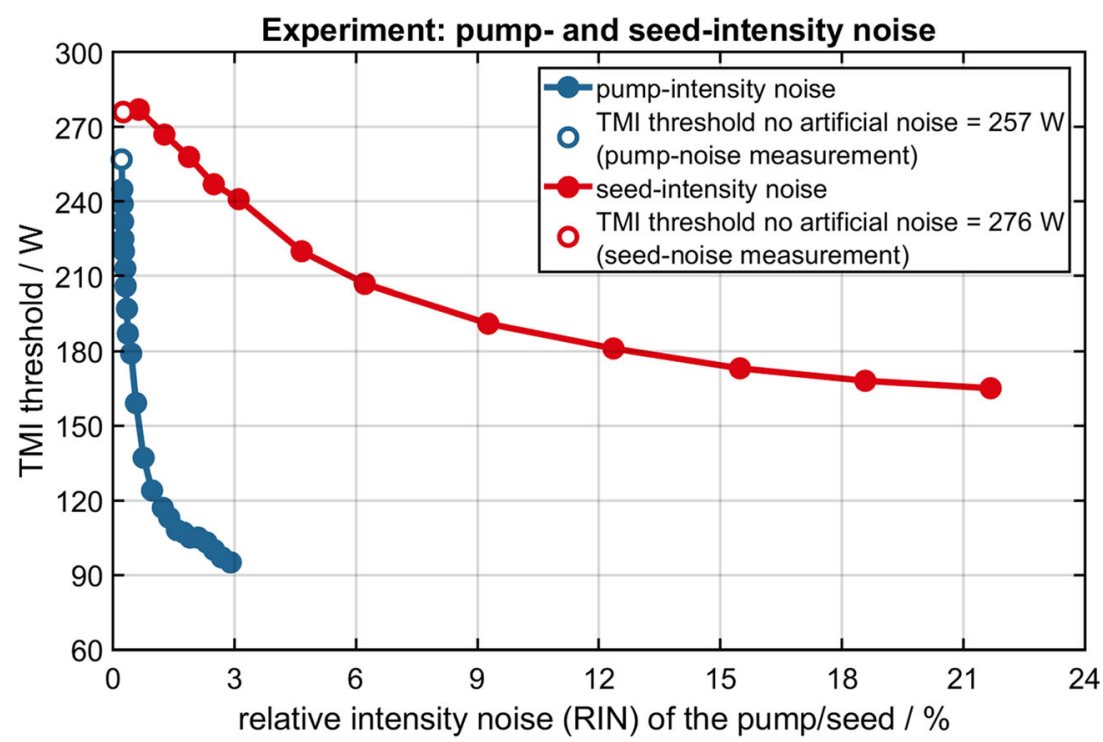

Fig. $7 \mathrm{TMl}$ threshold as a function of the relative intensity noise (RIN) of the pump and the seed. The artificially imprinted noise contains frequencies from $1 \mathrm{~Hz}$ up to $2 \mathrm{kHz}$. The impact of the pump intensitynoise (blue dots) on the TMI threshold is significantly stronger than that of the seed intensity-noise (red dots)

the frequency range around the main beam-fluctuation frequency of the fiber. In general, it can be concluded, that for high-power, large-mode-area fibers the pump intensity-noise below $20 \mathrm{kHz}$ should be reduced as much as possible.

\section{Seed intensity-noise}

As discussed earlier, the seed intensity-noise can also induce a phase shift between the MIP and the RIG by modulating the heat load in the fiber amplifier. Thus, the seed noise will in theory also be able to induce a modal energy transfer. Therefore, to unveil what mainly drives TMI, it is crucial to determine whether the seed or the pump intensity-noise has a stronger impact on the TMI threshold in high-power fiber amplifiers. With this purpose in mind, we used the same noise trace as for the pump intensity-noise and have imprinted it onto the seed radiation, as described in "Noise generation and experimental setup" section. The influence of the seed intensity-noise (measured at the fiber input) on the TMI threshold is depicted in Fig. 7. In particular, the figure illustrates the TMI threshold as a function of the seed-RIN (red dots) and compares this dependence to the RIN of the pump (blue dots: results from Fig. 6).

Note that, as already mentioned above, the initial TMI thresholds (i.e. when no artificial noise is applied; represented by the red and blue circles) of both measurements differ slightly, most likely due to maintenance-related adjustments in between the measurements. Nevertheless, as can be clearly seen from Fig. 7, the seed intensity-noise also causes a reduction of the TMI threshold in the same manner as the pump intensity-noise. However, at a seed-RIN of around 3\% the TMI threshold was decreased by just $13 \%$ from its intrinsic value ( $241 \mathrm{~W}$ vs. $276 \mathrm{~W}$ ), whereas for a similar pump intensity-noise the threshold was decreased by even $63 \%$ ( $95 \mathrm{~W}$ vs. $257 \mathrm{~W}$ ). Hence, the impact of pump intensity- 
noise on the TMI threshold is significantly stronger, which suggests that pump intensitynoise acts as the main driving force for TMI in saturated fiber amplifiers.

The experimental results obtained in this work can be now compared with former theoretical predictions. Smith et al. [11], Naderi et al. [12], Hansen et al. [13] and Tao et al. [14] all studied the influence of small seed modulations on the TMI threshold. All of them found that the seed modulations should lead to a decrease of the TMI threshold, which has been confirmed by our experimental observations. Moreover, all theoretical investigations identified the seed-modulation frequencies around the main frequency of the modal energy transfer (i.e. of the beam fluctuations) as the ones leading to the strongest decrease of the TMI threshold, which we have also verified experimentally in this work.

Additionally, the influence of small pump modulations on the TMI threshold was also studied and compared to the one of the seed modulations in [11, 14]. Tao et al. [14] described a similar impact of both noise sources on the TMI threshold, whereas Smith et al. [11] even predicted a stronger influence of the seed modulations. Both results are in contradiction to our experimental findings, which clearly show that the pump intensity-noise has the strongest impact on the TMI threshold. However, the experimental results presented in this work are in good agreement with our simulations and can be explained by already published theoretical and experimental work, as it will be detailed in the next section.

\section{Simulations and theoretical explanation}

By using the model presented in [19] and enabling mode coupling as demonstrated in [9], we have simulated the stability of a fiber amplifier at different pump- and seednoise amplitudes (RINs). To closely reproduce the experiments, the parameters of the simulated fiber, especially the mode-field diameter, have been adapted to those of the active fiber used in the experiments. Thus, the simulations used a step-index fiber with

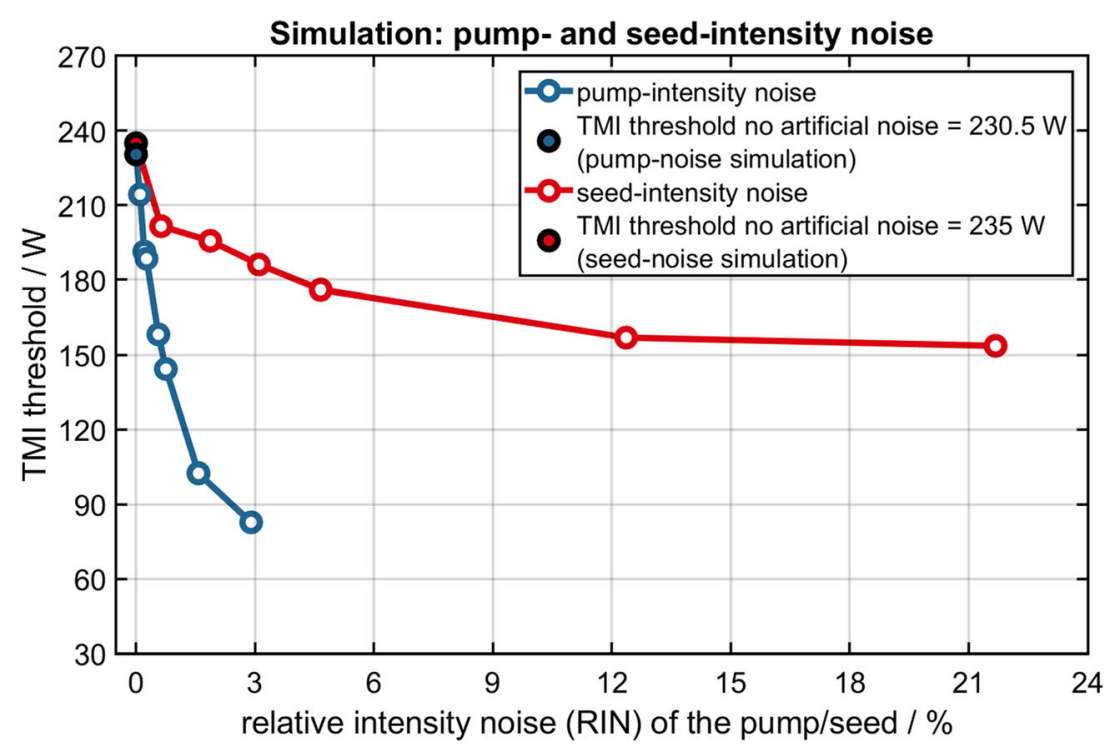

Fig. 8 Simulation results of the dependence of the TMI threshold on the relative intensity noise (RIN) of the pump (blue circles) and the seed (red circles). The simulations are in good agreement with the experimental results (Fig. 7) 
a mode-field diameter of $\sim 63 \mu \mathrm{m}(80 \mu \mathrm{m}$ core diameter), a V-parameter of 7 , a cladding diameter of $228 \mu \mathrm{m}$ and a length of $1 \mathrm{~m}$. Furthermore, the simulated noise trace was the one used in the experiments (however, limited to $50 \mathrm{~Hz} \ldots 20 \mathrm{kHz}$ due to the simulation time and resolution) and the noise amplitudes (RINs) in the simulations mostly reproduced the RIN levels of the experiments. Moreover, the fiber amplifier was seeded with an average power of $5 \mathrm{~W}$ at $1030 \mathrm{~nm}$ and pumped at $976 \mathrm{~nm}$ in the counter-propagating direction.

Our simulation model has one free parameter, which is the higher-order mode content at the fiber input. This is usually employed to calibrate the simulation results to the intrinsic TMI threshold of a fiber laser system. Additionally, in order to define the TMI threshold in the simulations, we decided to use the fluctuations (RIN) of the higher-order mode content as a sensitive measure for the modal energy transfer and, thus for the stability of the system. This strategy allowed for a significant reduction of the computation time. Thus, both the higher-order mode content and the RIN value for the threshold condition were adjusted to match the simulated TMI threshold to just one of our experimental points (in particular that corresponding to a seed-RIN of $21.7 \%$ ). This calibration procedure resulted in the selection of an input higher-order mode content of $7 \%$ for the simulations and a higher-order mode RIN of $25 \%$ as the TMI-threshold criterion. It is important to stress that, once these values were fixed, there were no free parameters left in our model. Additionally, it should be noted that the chosen TMI-threshold criterion of $25 \%$ higher-order mode RIN is in each case significantly above the output-signal RIN of the fiber, even for a high seed-input RIN since the latter is damped in the saturated fiber amplifier as will be detailed later. Thus, the calculated TMI thresholds are independent of the input noise, just like in the experiments. The simulation results for the pump and seed intensity-noise are illustrated in Fig. 8.

As can be seen, the simulations predict a non-linear behavior of the influence of the pump and the seed intensity-noise on the TMI threshold, which is in good agreement with the experimental observations. For the simulated pump-RIN (blue circles), the rapid decrease of the TMI threshold closely reproduces the experimental observations.
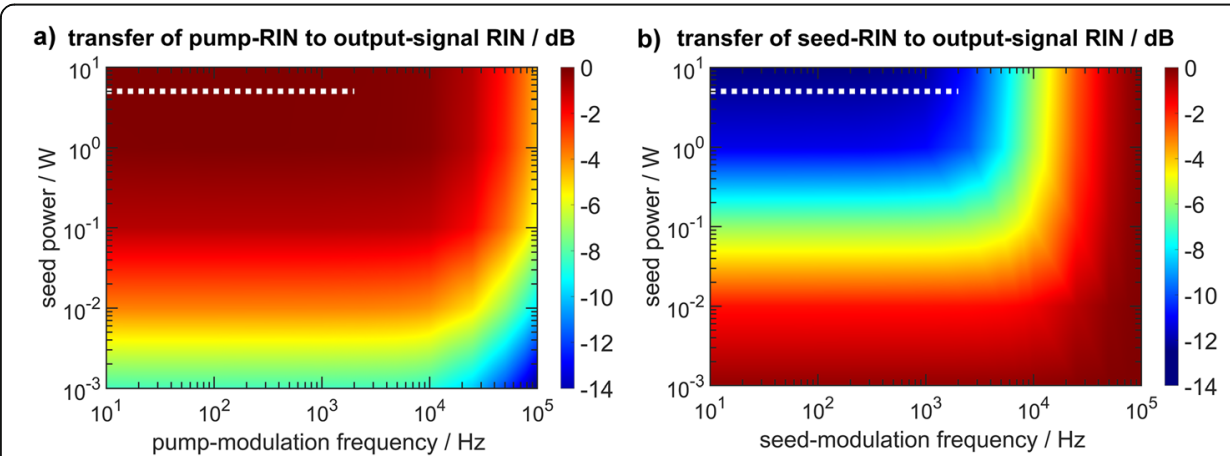

Fig. 9 Transfer function of the pump- and seed-RIN to the RIN of the output signal for an Yb-doped fiber amplifier as a function of the modulation frequency (x-axis) and the seed power (y-axis), adapted from [25]. a Transfer function of the pump-RIN: a low-pass behavior can be observed for a strong saturation (i.e. high seed power). $\mathbf{b}$ Transfer function of the seed-RIN: a high-pass behavior can be observed for a strong saturation (i.e. high seed power). The white dotted lines represent the frequency components $(1 \mathrm{~Hz} \ldots 2 \mathrm{kHz})$ and the average power $(5 \mathrm{~W})$ of the seed radiation used in the experiments and simulations presented in this work 
In particular, a pump-RIN of $2.9 \%$ resulted in a reduction of the TMI threshold of $64.1 \%$ from its intrinsic value in the simulations, which matches the drop of the TMI threshold in the experiments (63\%). Furthermore, also the seed-noise simulations (red circles) are consistent with the experiments. Particularly, at the highest seed-RIN of $21.7 \%$ the TMI threshold dropped by $34.6 \%$ in the simulations, which is in good agreement with the experimental threshold decrease of $40.2 \%$. Crucially, the simulations also predict that the pump intensity-noise should have a stronger impact on the TMI threshold than the seed intensity-noise, as can be seen in Fig. 8.

The experimental and theoretical findings of this work can be explained with the results presented in former studies [20-26], which have investigated the transfer functions of input pump- and seed-modulations to the modulations of the output-signal power in fiber amplifiers. All of these studies coincide in the same statement: the relative pump intensity-modulations in saturated fiber amplifiers are transferred to relative output-signal modulations following a low-pass behavior in the frequency domain. In contrast, the relative seed intensity-modulations experience a high-pass frequency filtering when being transferred to relative output-signal modulations in saturated fiber amplifiers. This behavior is exemplary illustrated in Fig. 9 for an Yb-doped fiber amplifier (adapted from [25]). In particular, Fig. 9a represents the transfer function of the pump-RIN to the RIN of the output signal, whereas Fig. 9b shows the transfer function of the seed-RIN to the RIN of the output signal, both as a function of the modulation frequency ( $x$-axis) and the seed power (y-axis). The simulations depicted in Fig. 9 were carried out for a $1.2 \mathrm{~m}$ long step-index fiber with a core diameter of $80 \mu \mathrm{m}(\sim 63 \mu \mathrm{m}$ mode-field diameter), a constant pump power of $300 \mathrm{~W}$ at $976 \mathrm{~nm}$ and an input RIN of 1\%. The white dotted lines in Fig. 9 represent the frequency components $(1 \mathrm{~Hz} \ldots 2 \mathrm{kHz})$ and the average power $(5 \mathrm{~W})$ of the seed radiation used in the experiments and simulations described in our work.

The following paragraph will focus just on the relevance of the noise transfer functions for TMI. However, a detailed description of their behavior and their physical origin can be found in the Appendix.

With the knowledge of the noise transfer functions it is possible to understand the weaker impact of the seed intensity-noise on the TMI threshold in saturated fiber amplifiers. As can be inferred from Fig. 9, the noise frequencies that influence the TMI threshold $(<2 \mathrm{kHz}$ for the used fiber, and in general $<20 \mathrm{kHz}$ for high-power fiber amplifiers) experience a different suppression in saturated fiber amplifiers (i.e. at high seed powers), depending on whether they are imprinted on the pump or the seed radiation. Thus, RIN of the pump with frequencies up to $20 \mathrm{kHz}$ is directly transferred to the RIN of the output signal without any significant attenuation at high seed powers (see upper part of Fig. 9a). On the contrary, RIN of the seed in the same frequency range is strongly damped when being transferred to the RIN of the signal output power in a saturated fiber amplifier (see upper part of Fig. 9b). Consequently, the signal output power of the fiber amplifier is significantly less modulated when noise with frequencies below $20 \mathrm{kHz}$ is imprinted on the seed radiation than when it is imprinted on the pump radiation. Therefore, the pump intensity-noise should have a stronger impact on the TMI threshold than the seed intensity-noise. This is because the strength of these outputsignal modulations determines the heat-load change in the fiber and, thereby, the resulting phase shift between the MIP and the RIG. As mentioned before, this phase 
shift can eventually induce a modal energy transfer at average powers below the intrinsic TMI threshold [10]. Hence, since pump intensity-noise below $20 \mathrm{kHz}$ generates stronger phase shifts than seed intensity-noise, pump-RIN should provoke a modal energy transfer at lower average powers than seed-RIN.

The results of our experimental investigations and simulations depict the described behavior and, thus, they are in good agreement with the physical understanding that has been revealed by the studies on the noise transfer in fiber amplifiers [20-26].

\section{Conclusions}

In this work, we have experimentally investigated the impact of pump and seed intensity-noise on the TMI threshold of a saturated high-power fiber amplifier. Furthermore, we have been able to closely reproduce the experimental results with our simulations. The experiments have been conducted by artificially imprinting noise onto the pump and the seed radiation, respectively. Thus, it has been found that both the pump and the seed intensity-noise result in a substantial decrease of the TMI threshold, since they both generate phase shifts between the modal interference pattern and the refractive index grating in the fiber and, herewith, induce a modal energy transfer. Therefore, the strong influence of both noise sources must be taken into account when comparing TMI thresholds between different fiber laser systems. This finding emphasizes, that the TMI threshold is not only determined by the active fiber itself, but it is defined by the whole system (including e.g. the pump and the seed source). Furthermore, it has been observed that noise frequencies around the main beam-fluctuation frequency of the fiber (e.g. $125 \ldots 500 \mathrm{~Hz}$ for the used LPF) have the strongest influence on the TMI threshold.

Most importantly, the investigations have revealed that the pump intensity-noise has a significantly stronger impact on the TMI threshold in saturated fiber amplifiers than the seed intensity-noise. In the experiments a pump intensity-noise of only $2.9 \%$ led to a strong reduction of the TMI threshold of $63 \%$ from its intrinsic value, whereas a similar seed-noise level reduced it by just $13 \%$. This, as has been discussed, is because the TMI-relevant pump-noise frequencies $(<20 \mathrm{kHz})$ are transferred to the signal output power without significant attenuation in a saturated $\mathrm{Yb}$-doped fiber amplifier. Thus, they directly induce phase shifts and, by that, enable an energy transfer between different transverse modes. In contrast, seed-noise frequencies below $20 \mathrm{kHz}$ are strongly attenuated in saturated fiber amplifiers and, thus, their contribution to phase shifts is weakened. Consequently, pump intensity-noise has a stronger impact on the TMI threshold and can be considered as the main driving force for TMI in saturated fiber amplifiers.

The presented findings are not limited to a specific fiber type, but they are valid for saturated Yb-doped fiber amplifiers in general, since all these systems have similar noise transfer functions. Moreover, the pump intensity-noise will most likely also influence the TMI threshold in high-power fiber oscillators, since the underlying physics of the phase-shift introduction is similar. However, no investigations on this dependence exist so far.

Furthermore, our investigations have shown a steep increase of the TMI threshold for low pump-noise levels, which indicates that a reduction of this noise around the 
main beam-fluctuation frequency could lead to a significant increase of the TMI threshold. Thus, reducing the noise of the pump-diode/laser-driver system below $20 \mathrm{kHz}$ represents a promising approach to mitigate TMI in high-power fiber laser systems. Additionally, when the pump intensity-noise has been sufficiently suppressed in the frequency region of interest, a reduction of the seed intensity-noise could lead to a further increase of the TMI threshold.

\section{Appendix}

\section{Noise transfer functions}

In this section the noise transfer functions for pump- and seed-RIN in fiber amplifiers are described in detail and their physical origin is explained. This is done with the help of Fig. 9 (adapted from [25]), which illustrates the transfer of relative pump intensitymodulations (Fig. 9a) and relative seed intensity-modulations (Fig. 9b) to relative intensity modulations of the output signal in an $\mathrm{Yb}$-doped fiber amplifier. The noise transfer is depicted as a function of the modulation frequency ( $x$-axis) and the seed power ( $y$ axis). These simulations used a $1.2 \mathrm{~m}$ long step-index fiber with a core diameter of $80 \mu \mathrm{m}$ ( $63 \mu \mathrm{m}$ mode-field diameter), a constant pump power of $300 \mathrm{~W}$ at $976 \mathrm{~nm}$ and an input RIN of $1 \%$.

As can be seen in Fig. 9a, for saturated fiber amplifiers (i.e. high seed powers) the relative pump intensity-noise with frequencies up to $20 \mathrm{kHz}$ is directly transferred to the relative output-signal noise without suffering any significant attenuation. However, higher frequencies are attenuated, which results in the low-pass character of the transfer function of pump-RIN to output-signal RIN. This frequency dependence is related to the effective lifetime of the upper laser level [25]. Thus, pump modulations with frequencies lower than the inverse effective lifetime (i.e. slow modulations) are able to directly change the inversion in the fiber and, consequently, the gain and the signal output power. In contrast, if the pump modulation has a frequency higher than the inverse of the effective lifetime (i.e. a fast modulation), the inversion cannot follow the pump intensity-change and, thus, the pump intensity-noise is not fully transferred to the signal output power, i.e. it is damped.

For low seed powers (i.e. with low saturation), the transfer of pump intensity-noise to the signal output power is damped in the complete frequency range, albeit higher frequencies still suffer a stronger attenuation (see lower part of Fig. 9a). This is because at low seed powers a specific relative pump intensity-change (RIN) has a weaker influence on the gain (due to the higher inversion level) and, thus, on the signal output power than at high seed powers.

It is interesting to see in Fig. 9b that the transfer function of the relative seed intensity-noise to the relative noise of the output signal in saturated fiber amplifiers (i.e. at high seed powers) has the opposite behavior than for the pump intensity-noise. This means that, in the case of seed noise, frequencies below $20 \mathrm{kHz}$ are strongly damped, whereas higher noise frequencies suffer a lower attenuation. In other words, the transfer function exhibits a high-pass filtering behavior. Again, this frequency dependence is a consequence of the effective lifetime of the upper laser level. Thus, seed modulations with frequencies lower than the inverse of the effective lifetime (i.e. slow modulations) are able to modify the inversion in the fiber, whereas seed modulations with frequencies higher than the inverse of the effective lifetime (i.e. fast modulations) 
cannot. The reason why this behavior leads to a high-pass filtering of the seed modulations is the following: in the case of slow seed modulations, a seed intensity-peak will decrease the inversion and, thus, also the gain, which directly attenuates the noise peak (relative to the average signal power). A slow seed intensity-dip will result in an increased inversion and, thus, in an increased gain that, in turn, will reduce the depth of the dip (relative to the average signal power). Consequently, slow seed modulations are attenuated relative to the average signal power in a saturated fiber amplifier. In contrast, fast seed intensity-modulations (i.e. with frequencies higher than the inverse of the effective lifetime) do not significantly affect the inversion/gain of the fiber, which leads to a constant amplification of seed peaks and dips along the fiber amplifier, resulting in an undamped transfer of the seed-RIN to the output-signal RIN.

At low seed powers (i.e. with low saturation) the seed intensity-noise suffers a lower overall attenuation (see lower part of Fig. 9b) because here a change in the seed power is not able to strongly affect the inversion/gain of the fiber amplifier (due to the low saturation). Hence, the seed modulations are not attenuated but constantly amplified. This results in an undamped transfer of the seed-RIN to the RIN of the output signal, which is in contrast to the behavior at high saturation (i.e. at high seed powers).

\section{Abbreviations}

AWG: Arbitrary waveform generator; LPF: Large pitch fiber; MIP: Modal interference pattern; RIG: Refractive index grating; RIN: Relative intensity noise; TMI: Transverse mode instability

\section{Acknowledgements}

Not applicable.

\section{Authors' contributions}

CS and CJ designed and set up the experiments. CS and SK performed the experiments and analyzed the data. CS wrote the manuscript. CS, CJ and JL interpreted the data. All authors read and edited the manuscript and approved the final version.

\section{Funding}

Deutsche Forschungsgemeinschaft (DFG, German Research Foundation) - 416342637; 416342891; GRK 2101 (259607349). Fraunhofer Gesellschaft - Fraunhofer Cluster of Excellence "Advanced Photon Sources".

Availability of data and materials

The datasets used and/or analysed during the current study are available from the corresponding author on reasonable request.

Competing interests

The authors declare that they have no competing interests.

\section{Author details}

${ }^{1}$ Institute of Applied Physics, Abbe Center of Photonics, Friedrich-Schiller-Universität Jena, Albert-Einstein-Str. 15,07745 Jena, Germany. ${ }^{2}$ Fraunhofer Institute for Applied Optics and Precision Engineering, Albert-Einstein-Str. 7, 07745 Jena, Germany. ${ }^{3}$ Helmholtz-Institute Jena, Fröbelstieg 3, 07743 Jena, Germany.

Received: 29 November 2019 Accepted: 10 February 2020

Published online: 10 March 2020

\section{References}

1. Jauregui C, Limpert J, Tünnermann A. High-power fibre lasers. Nat Photonics. 2013;7:861-7.

2. Zervas MN, Codemard CA. High power fiber lasers: a review. IEEE J Sel Top Quantum Electron. 2014;20:1-23.

3. Eidam T, Hanf S, Seise E, et al. Femtosecond fiber CPA system emitting 830 W average output power. Opt Lett. 2010;35: 94-6.

4. Eidam T, Wirth $C$, Jauregui $C$, et al. Experimental observations of the threshold-like onset of mode instabilities in high power fiber amplifiers. Opt Express. 2011;19:13218-24.

5. Otto H-J, Stutzki F, Jansen F, et al. Temporal dynamics of mode instabilities in high-power fiber lasers and amplifiers. Opt Express. 2012;20:15710.

6. Jauregui C, Eidam T, Limpert J, Tünnermann A. Impact of modal interference on the beam quality of high-power fiber amplifiers. Opt Express. 2011;19:3258-71.

7. Erdogan T. Fiber grating spectra. J Lightwave Technol. 1997:15:1277-94.

8. Smith AV, Smith JJ. Mode instability in high power fiber amplifiers. Opt Express. 2011;19:10180-92. 
9. Stihler $C$, Jauregui $C$, Tünnermann A, Limpert J. Phase-shift evolution of the thermally-induced refractive index grating in high-power fiber laser systems induced by pump-power variations. Opt Express. 2018;26:19489-97.

10. Stihler $C$, Jauregui $C$, Tünnermann A, Limpert J. Modal energy transfer by thermally induced refractive index gratings in Yb-doped fibers. Light Sci Appl. 2018;7:59.

11. Smith AV, Smith JJ. Influence of pump and seed modulation on the mode instability thresholds of fiber amplifiers. Opt Express. 2012;20:24545-58.

12. Naderi S, Dajani I, Madden T, Robin C. Investigations of modal instabilities in fiber amplifiers through detailed numerical simulations. Opt Express. 2013;21:16111-29.

13. Hansen KR, Alkeskjold TT, Broeng J, Lægsgaard J. Theoretical analysis of mode instability in high-power fiber amplifiers. Opt Express. 2013;21:1944-71.

14. Tao $R$, Wang $X$, Zhou P. Comprehensive theoretical study of mode instability in high-power fiber lasers by employing a universal model and its implications. IEEE J Sel Top Quantum Electron. 2018;24:1-19.

15. Limpert J, Stutzki F, Jansen F, et al. Yb-doped large-pitch fibres: effective single-mode operation based on higher-order mode delocalisation. Light Sci Appl. 2012;1:e8.

16. Beier F, Möller F, Sattler B, et al. Experimental investigations on the TMl thresholds of low-NA Yb-doped single-mode fibers. Opt Lett. 2018;43:1291.

17. Johansen MM, Laurila M, Maack MD, et al. Frequency resolved transverse mode instability in rod fiber amplifiers. Opt Express. 2013;21:21847-56.

18. Ward B, Robin C, Dajani I. Origin of thermal modal instabilities in large mode area fiber amplifiers. Opt Express. 2012;20: $11407-22$.

19. Jauregui C, Stihler C, Tünnermann A, Limpert J. Pump-modulation-induced beam stabilization in high-power fiber laser systems above the mode instability threshold. Opt Express. 2018;26:10691-704.

20. Novak S, Moesle A. Analytic model for gain modulation in EDFAs. J Lightwave Technol. 2002;20:975-85.

21. Tröbs M, Weßels P, Fallnich C. Power- and frequency-noise characteristics of an Yb-doped fiber amplifier and actuators for stabilization. Opt Express. 2005;13:2224.

22. Tünnermann $\mathrm{H}$, Neumann J, Kracht $\mathrm{D}$, Weßels $\mathrm{P}$. Gain dynamics and refractive index changes in fiber amplifiers: a frequency domain approach. Opt Express. 2012;20:13539-50.

23. Steinke M, Neumann J, Kracht D, Wessels P. Gain dynamics in Er^3+:Yb^3+ co-doped fiber amplifiers. Opt Express. 2015; 23:14946.

24. Zhao J, Guiraud G, Floissat F, et al. Gain dynamics of clad-pumped Yb-fiber amplifier and intensity noise control. Opt Express. 2017;25:357.

25. Jauregui C, Müller M, Kienel M, et al. Optimizing the noise characteristics of high-power fiber laser systems. Proc SPIE. 2017;10083:100830W.

26. Gierschke P, Jauregui C, Gottschall T, Limpert J. Relative amplitude noise transfer function of an $\mathrm{Yb}^{3+}$-doped fiber amplifier chain. Opt Express. 2019;27:17041.

\section{Publisher's Note}

Springer Nature remains neutral with regard to jurisdictional claims in published maps and institutional affiliations.

\section{Submit your manuscript to a SpringerOpen ${ }^{\circ}$ journal and benefit from:}

- Convenient online submission

- Rigorous peer review

- Open access: articles freely available online

- High visibility within the field

- Retaining the copyright to your article

Submit your next manuscript at $\boldsymbol{\nabla}$ springeropen.com 